\title{
The Effects of Grape Seed Extract Ointment on Approximation of Cesarean Section Wound
}

\author{
Sima Soorgi, ${ }^{1}$ Ali Mohammad Izadpanah, ${ }^{2,}{ }^{*}$ Gholam Reza Sharifzadeh, ${ }^{3}$ Marziyeh Torshizi, ${ }^{2}$ Zohreh \\ Khazaye, ${ }^{4}$ and Mohammad Hasanpour ${ }^{5}$ \\ ${ }^{1}$ Faculty of Nursing and Midwifery, Birjand University of Medical Sciences, Birjand, IR Iran \\ ${ }^{2}$ East Nursing and Midwifery Research Center, Birjand University of Medical Sciences, Birjand, IR Iran \\ ${ }^{3}$ Social Determinants of Health Research Center, Birjand University of Medical Sciences, Birjand, IR Iran \\ ${ }^{4}$ Department of Gynecology, Faculty of Medicine, Birjand University of Medical Sciences. Birjand, IR Iran \\ ${ }^{5}$ Department of Physiology and Pharmacology, Faculty of Medicine, Birjand University of Medical Science, Birjand, IR Iran \\ "Corresponding author: Ali Mohammad Izadpanah, Instructor of Nursing, East Nursing and Midwifery Research Center, Faculty of Nursing and Midwifery, Birjand University \\ of Medical Sciences, Birjand, IR Iran. Tel: +98-5632381416, E-mail: izadpanahy@yahoo.com
}

Received 2015 August 05; Revised 2015 December 06; Accepted 2015 December 23.

\begin{abstract}
Background: Cesarean section is among the most important gynecological surgeries and is the most common surgery in the present era. Delayed wound healing is among the main post-cesarean complications. It dramatically affects female's quality of life and causes frequent medical visits and even re-hospitalization.

Objectives: This study aimed at investigating the effects of grape seed extract on the approximation of cesarean section wound. Methods: This double-blind randomized controlled trial was done during year 2015 on a convenient sample of 129 eligible females, who had undergone a cesarean section at Valiasr hospital, Birjand, Iran. The candidates were randomly allocated to a placebo and two experimental groups. In one experimental group, the participants were treated with $2.5 \%$ grape seed extract ointment (group A) while in the other, $5 \%$ ointment was used (group B). Wound approximation was measured by using the redness, edema, ecchymosis, discharge, approximation (REEDA) scale at three time points including before ointment application (T1) as well as at the sixth (T2) and the fourteenth (T3) post-intervention days. The data were analyzed via the SPSS (v. 19.0) software and through analysis of variance, Chi-square, Fisher's exact, Friedman's, Wilcoxon, Kruskal-Wallis and Mann-Whitney U tests, at a significance level of 0.05. Results: Before the study intervention, the difference among the groups regarding the mean score of wound approximation was not statistically significant $(\mathrm{P}=0.14)$. However, there were significant differences among the groups regarding the mean score of wound approximation at $\mathrm{T} 2(\mathrm{P}=0.002)$ and $\mathrm{T} 3(\mathrm{P}=0.02)$.

Conclusions: The findings of this study demonstrate the effectiveness of grape seed extract ointment in accelerating cesarean wound closure. Therefore, the ointment can be used to accelerate wound healing after cesarean section.
\end{abstract}

Keywords: Grape Seed Extract, Cesarean Section, Wound Healing

\section{Background}

Cesarean Section (CS) is among the most common gynecological surgeries. The rate of CS is progressively increasing due to factors such as increased age of marriage, people's socio-economic status, and female's gynecological problems (1). According to the World Health Organization, the optimum rate of CS is $15 \%$. Nonetheless, $10 \%-30 \%$ of all deliveries around the world are currently performed through CS (2). Statistics also show that after Brazil, Cyprus and Columbia, Iran is the fourth country in terms of CS rate (3). About $50 \%$ to $60 \%$ of all deliveries (i.e. one out of two deliveries) in Iran are performed through CS (2).

There are factors, which can increase the risk for developing CS-related complications, including wound site infection, wound dehiscence, necrotizing fasciitis, peritonitis, scars, and prolonged hospital stay (4). They include dia- betes mellitus, hypertension, lengthy membrane rupture, emergency CS, giving birth to twins (5), lengthy labor or CS (6), corticosteroid therapy (7), immunosuppression, anemia, poor hemostasis and hematoma formation (8). Poor wound healing and surgical site dehiscence are two major factors behind post-CS complications (9). While bearing their maternal responsibilities, females, who undergo CS, usually experience pain, discomfort and surgical site complications. Such complications delay initiation of breastfeeding and cause early neonatal weight loss (8).

Many pharmacological and non-pharmacological interventions can be used to accelerate the wound healing process. However, despite their high efficacy, synthetic mediations are usually associated with different side effects (10). Therefore, herbal extracts such as grape seed, lemon, green tea and rosemary extracts are used to accel- 
erate wound healing. The common characteristic of all these plants is the production of a compound with phenolic structure (11).

Rich in phenolic compounds such as catechin, epicatechin, and dimeric, trimeric and tetrameric proanthocyanidins (12), grape seeds are widely used in the management of many illnesses such as cancers, cardiovascular disease, gastric ulcers, severe obesity and skin inflammations (13). Besides phenolic compounds, grape seed also contains vitamin $\mathrm{E}$ (a strong reductive agent), which helps preserve skin moisture and accelerates wound healing (14). Proanthocyanidins in the grape seed enclose collagen and elastin, protect them against enzymes, and promote the development of elastin and collagen tissues (14). As antioxidants, proanthocyanidins also potentially induce vascular endothelial growth factor in human cells (15) and thereby, accelerate wound healing, reduce edema and encourage blood flow (14).

Accelerating the process of wound healing has many economical and health-related benefits; for example, the faster the wound healing, the lesser the probability of wound infection (16). Wound infection, which is associated with hematoma, serous secretions and dehiscence, is among the most common causes of post-CS deaths. Such an infection also causes frequent hospitalizations or medical visits for wound debridement, secretion drainage and wound care, which in turn increase healthcare costs (17). Besides, delayed healing of CS surgical wound can affect mother-child relationships, cause anxiety, discomfort, stress and dissatisfaction for mothers, and impose heavy financial and emotional burdens on mothers and communities (8).

\section{Objectives}

Given the progressively increasing rate of CS and CS wound complications as well as the importance of CS wound healing, this study was undertaken to investigate the effects of grape seed extract on the approximation of CS wound.

\section{Methods}

This double-blind randomized controlled trial was done in 2015 on 129 eligible females, who had undergone a CS at Valiasr hospital, Birjand, Iran. Based on the findings reported by Sehhati Shaffaie et al. (2012) (18), the results of the sample size calculation formula for comparing the two means revealed that 36 participants were needed for each study group. However, in order to improve the rigor of the study and also to compensate a probable attrition of $20 \%$, the size of the necessary sample for each study group was determined to be 43 . Eligible females were recruited conveniently and were randomly allocated to a placebo and two experimental groups. In one experimental group, the females were treated with $2.5 \%$ grape seed extract ointment (group A) while in the other, 5\% ointment was used (group B).

Females were included if they were in the 37th to 42nd week of their pregnancy, had undergone a transverse CS; had an age of 15 to 35, a body mass index (BMI) of 18.5 to 25 , a singleton pregnancy, and an Iranian ethnicity; had no history of acute or chronic conditions, drug abuse, active smoking, or disorders affecting wound healing (such as diabetes mellitus, anemia, or renal, hepatic and mental disorders); had no allergy to grape seed extract ointment; had not undergone a previous CS; and had no history of using drugs, which could affect wound healing (like glucocorticoids, anticoagulants, immunosuppressant medications, antibiotics and chemotherapy agents). The exclusion criteria included using the ointment irregularly, developing complications associated with long-term use of the ointment, developing wound infection, and making changes in their antibiotic medications.

The grape species, Vitis vinifera L. from the Vitaceae family, was identified by botanists affiliated to Birjand Unviersity, Birjand, Iran. Then, its seeds were separated and sent to Dineh Iran pharmaceutical company in order to produce $2.5 \%$ and $5 \%$ ointments. In the company, the seeds were washed, dried and grinded by an electric mill. Then, the seed extract was taken through hydroalcoholic extraction procedure, using 70\% alcohol as follows. Primarily, $1050 \mathrm{~mL}$ of $70 \%$ alcohol was added to $50 \mathrm{~g}$ of grape seed powder. Then, it was mixed by using a mixer and kept in an oven for 24 hours at $40 \pm 2^{\circ} \mathrm{C}$. Thereafter, the mixture was sifted. The residue was then mixed with $450 \mathrm{~mL}$ of $70 \%$ alcohol, kept in the oven for 24 hours at $40 \pm 2{ }^{\circ} \mathrm{C}$, and sifted. All extracts were dried at $50^{\circ} \mathrm{C}$ by using an evaporator. This process was undertaken five times in order to produce 99 $\mathrm{g}$ of hydroalcoholic grape seed extract. Finally, $30 \mathrm{~g}$ ointment tubes containing either placebo or grape seed ointment were produced and numbered with random numbers from 2 to 130. Numbering was performed by a pharmacist, who had produced the ointments, and thus the researchers were blind to the treatments. One ointment tube plus a teaspoon (with a capacity of $0.9 \mathrm{~g}$ of the ointment) was given to each participant. For each topical use of the ointment, the candidates were asked to wash their hands thoroughly and then apply a spoonful of the ointment ( $1 \mathrm{~g}$ ) to their CS wounds and sutures two times a day (i.e. each twelve hours) from the second to the fifteenth post-CS days. We made telephone contacts with the participants in order to assess and emphasize ointment use. They 
were recommended to wear cotton shirts, take a shower after hospital discharge and on every other day, remain physically active, avoid lifting heavy loads and eating flatulent foods, avoid doing any movements, which could cause excessive stretching of the sutures, support their abdomen with their hands when coughing, use emollient foods in order to prevent constipation, and receive dairy products, meat, fruits and vegetables on a daily basis.

A metal ruler, the redness, edema, ecchymosis, discharge, approximation (REEDA) scale, and a questionnaire on the female's personal and CS-related characteristics were used to gather data. The REEDA is an international scale for assessing CS wound. It is comprised of five items of redness, edema, ecchymosis, discharge and approximation, which are scored from 0 to 3 , resulting in a total REEDA score of 0 to 15.

The dressing of the CS wound was removed 48 hours after CS, i.e. at hospital discharge. Wound condition was assessed three times including at dressing removal and before applying ointment (T1) as well as on the sixth (T2) and the fourteenth (T3) post-intervention days. Assessments were performed by the first author, who was blind to the treatments, and by using a metal ruler and the REEDA scale. During wound assessment, patients were asked to lie in the supine position.

Primarily, the number of females in each study group was 43. However, four patients were excluded during the course of the study. Therefore, data analysis was done on data retrieved from 125 females (41 in experimental group A, 42 in experimental group B, and 42 in the placebo group). The data were analyzed via the SPSS (v. 19.0) software and by perfoming analysis of variance (ANOVA), Chisquare, Friedman, Wilcoxon, Kruskal-Wallis, and MannWhitney $U$ tests at a significance level of 0.05 .

This study was carried out after obtaining approval from the Ethics Committee of Birjand University of Medical Sciences (with the code of IR.BUMS.1393.6) and registration in the Iranian Registry of Clinical trials (with the code of IRCT2014122220393N1).

\section{Results}

There were no significant differences among the groups considering the participants' age (P 0.94), educational status $(\mathrm{P}=0.16)$, BMI at the beginning of pregnancy $(P=0.74)$, number of pregnancies $(P=0.87)$, number of deliveries $(P=0.28)$, length of $C S$ incision $(P=0.71)$, the cause for CS $(\mathrm{P}=0.99)$, type of anesthesia for CS $(\mathrm{P}=0.41)$, and history of previous CS $(\mathrm{P}=0.98)$ (Tables 1 and 2 ).

Between-group comparisons revealed that the groups did not differ significantly from each other in terms of the pretest values of CS wound approximation $(\mathrm{P}=0.14)$.
However, the differences among the groups regarding CS wound approximation at $\mathrm{T} 2$ and T3 were statistically significant $(\mathrm{P}=0.0002$ and 0.02 , respectively). Post hoc analysis showed that at T2, CS wound approximation in experimental group $B(0.69 \pm 0.5)$ was significantly greater than experimental group $\mathrm{A}(0.9 \pm 0.3)(\mathrm{P}=0.02)$ and the placebo group $(0.95 \pm 0.2)(\mathrm{P}=0.002)$. Besides, CS wound approximation in experimental group B at T3 $(0.50 \pm 0.2)$ was also greater than experimental group $\mathrm{A}(0.24 \pm 0.4)(\mathrm{P}=0.01)$ and the placebo group $(0.26 \pm 0.4)(\mathrm{P}=0.007)$. However, CS wound approximation in experimental group A was not significantly different from the placebo group neither at $\mathrm{T} 2$ $(\mathrm{P}=0.38)$ nor at T3 $(\mathrm{P}=0.85)$ (Table 3$)$.

\section{Discussion}

The findings of the present study showed that the difference among the groups regarding CS wound approximation was not statistically significant at $\mathrm{T} 1(\mathrm{P}=0.14)$. However, at T2 $(\mathrm{P}=0.002)$ and T3 $(\mathrm{P}=0.02)$, the differences were statistically significant.

Hemmati et al. (2014) conducted a double-blind clinical trial to assess the effects of $2 \%$ topical cream of grape seed on surgical wound healing. They recruited 40 patients from Imam Khomeini skin care clinic, Ahvaz, Iran, and randomly allocated them to a treatment and a placebo group. Then, the patients underwent minor surgeries for skin tags and moles located on different parts of the body except for the face. They asked their participants to apply one centimeter of grape seed cream on their surgical wounds twice a day. Their findings showed that in the intervention and placebo groups, complete wound healing occurred, respectively, on the eighth and the fourteenth post-surgery days, respectively. Their statistical analyses showed a significant difference between the groups regarding the time of achieving recovery $(\mathrm{P}<0.001)(19)$. In line with our findings, their findings confirmed the positive effects of grape seed extract on wound healing. In another study, Hemmati et al. (2011) also found that $2 \%$ grape seed extract accelerated the process of wound contraction and closure and reduced wound healing time from 20 to 13 days (20). Nayak et al. (2010) also assessed the effects of grape skin powder on full-thickness skin lesions in mice and found that complete wound contraction and healing occurred thirteen days after the beginning of their treatment (21). The findings of all these studies confirm our findings.

Khanna et al. (2001) also performed an experimental study to assess the effects of proanthocyanidin extract of grape seed on in vivo wound healing among nine male mice (BalbC) aged four to six weeks. Their findings revealed 
Table 1. The Frequency Distribution of the Study Participants in Terms of Their Demographic Characteristics ${ }^{\mathrm{a}}$

\begin{tabular}{|c|c|c|c|c|}
\hline Groups/Characteristics & $5 \%$ ointment $(N=42)$ & $2.5 \%$ ointment $(\mathrm{N}=41)$ & $\operatorname{Placebo}(\mathrm{N}=42)$ & PValue \\
\hline Educational status & & & & 0.16 \\
\hline Illiterate & 0 & $5(12.2)$ & $1(2.38)$ & \\
\hline Primary & $10(23.8)$ & $10(24.4)$ & $12(28.58)$ & \\
\hline Guidance school & $10(23.8)$ & $8(19.5)$ & $4(9.53)$ & \\
\hline High school & $13(31)$ & $8(19.5)$ & $13(30.96)$ & \\
\hline University & $9(21.4)$ & $10(24.4)$ & $12(28.58)$ & \\
\hline Reason behind CS & & & & 0.99 \\
\hline Previous CS & $17(40.5)$ & $18(43.9)$ & $18(42.86)$ & \\
\hline Fetal reasons & $19(45.2)$ & $18(43.9)$ & $18(42.86)$ & \\
\hline Maternal reasons & $6(14.3)$ & $5(12.2)$ & $6(14.29)$ & \\
\hline Type of anesthesia & & & & 0.41 \\
\hline General & $22(52.4)$ & $28(68.3)$ & $21(50)$ & \\
\hline Spinal & $19(45.2)$ & $12(29.3)$ & $19(45.2)$ & \\
\hline Both & $1(2.4)$ & $1(2.4)$ & $2(4.8)$ & \\
\hline Previous history of CS & & & & 0.98 \\
\hline Yes & $19(45.2)$ & $19(46.3)$ & $20(47.6)$ & \\
\hline No & $23(54.8)$ & $22(53.7)$ & $22(52.4)$ & \\
\hline
\end{tabular}

${ }^{\mathrm{a}}$ Values are expressed as No. (\%).

Table 2. Comparing Groups Regarding the Participants' Demographic Characteristics ${ }^{\mathrm{a}}$

\begin{tabular}{|c|c|c|c|c|}
\hline Groups Characteristics & $5 \%$ Ointment $(\mathrm{N}=42)$ & 2.5\% Ointment $(\mathrm{N}=41)$ & Placebo $(\mathrm{N}=\mathbf{4 2})$ & P Value \\
\hline Age, $y$ & $28.3 \pm 5.2$ & $28.7 \pm 5$ & $28.6 \pm 4.6$ & 0.94 \\
\hline BMI at the beginning of pregnancy, $\mathrm{kg} / \mathrm{m}^{2}$ & $21.8 \pm 2.1$ & $21.5 \pm 2$ & $21.7 \pm 1.9$ & 0.74 \\
\hline Number of pregnancies & $2.5 \pm 1.5$ & $2.6 \pm 1.7$ & $2.7 \pm 1.6$ & 0.87 \\
\hline Number of deliveries & $2.2 \pm 1.2$ & $2.5 \pm 1.5$ & $2 \pm 0.9$ & 0.28 \\
\hline Length of CS incision, $\mathrm{cm}$ & $13.96 \pm 1.03$ & $14.05 \pm 1.06$ & $13.87 \pm 0.92$ & 0.71 \\
\hline
\end{tabular}

${ }^{\mathrm{a}}$ Values are expressed as mean $\pm \mathrm{SD}$

Table 3. Comparing the Groups Regarding Cesarean Section Wound Approximation at Different Measurement Time Points

\begin{tabular}{|c|c|c|c|c|}
\hline Groups/Characteristics & $5 \%$ Ointment $(\mathrm{N}=42)$ & 2.5\% Ointment $(\mathrm{N}=41)$ & Placebo $(N=42)$ & $\begin{array}{l}\text { P Value (the Kruskal-Wallis and } \\
\text { the Mann-Whitney U Tests) }\end{array}$ \\
\hline \multicolumn{5}{|l|}{ Wound approximation, $\mathrm{mm}$} \\
\hline Before & $1.05 \pm 0.2$ & $1.0 \pm 0.0$ & $1.0 \pm 0.0$ & $\mathrm{P}=0.14$ \\
\hline $\begin{array}{l}\text { Six days after the } \\
\text { intervention }\end{array}$ & $0.69 \pm 0.5$ & $0.90 \pm 0.3$ & $0.95 \pm 0.2$ & $\begin{array}{c}\mathrm{P}=0.002,2.5 \% \text { - } 5 \% \text { comparison: } \\
0.02 ; 5 \% \text {-Placebo comparison: } \\
0.002\end{array}$ \\
\hline $\begin{array}{l}\text { Fourteen days after the } \\
\text { intervention }\end{array}$ & $0.05 \pm 0.2$ & $0.24 \pm 0.4$ & $0.26 \pm 0.4$ & $\begin{array}{c}\mathrm{P}=0.02,2.5 \%-5 \% \text { comparison: } 0.01 \\
, 5 \%-\text { Placebo comparison: } 0.007\end{array}$ \\
\hline $\begin{array}{l}\text { The results of the Friedman's } \\
\text { test }\end{array}$ & $X^{2}=63.74 ; \mathrm{df}=2, P<0.001$ & $X^{2}=55.03, d f=2, P<0.001$ & $X^{2}=58.26 ; \mathrm{df}=2 ; P<0.001$ & - \\
\hline
\end{tabular}

that topical use of grape seed extract, containing 5000PPM resveratrol, accelerated wound contraction and closure $(\mathrm{P}<0.05)$. They also found that treatment with this extract was associated with well-defined hyper-proliferative epithelial region, higher cell density, enhanced deposition of connective tissue, and improved histological structure. Besides, the extract promoted the production of vascular endothelial growth factor at the wound edge. They concluded that topical use of grape seed ointment facilitates wound healing (22). 
Bastami et al. (2014) also investigated the effects of phenytoin on CS wound healing and found that on the seventh and the fourteenth days of their intervention, wound healing in their experimental group was significantly better than their control group $(P=0.001)$ (23). The design and the methods of their study were similar to the present study and their findings revealed that phenytoin could accelerate CS wound healing.

Heydari et al. (2013) also performed a double-blind prospective randomized clinical trial in Arak, Iran, in order to evaluate the effects of honey (Iranian Astragalus gossypinus) on CS wound and scar. They allocated 130 eligible females to a honey therapy, a placebo, and a control group. In contrary to our findings, which indicated the effectiveness of grape seed extract ointment in promoting wound healing, their findings revealed that on the tenth and the 40th post-CS days, there was no significant difference among their groups in terms of CS wound healing (8).

Sehhati Shaffaie et al. (2012) also investigated the effects of phenytoin cream on episiotomy wound healing and found that on the first and the tenth post-CS days, wound healing mean score in their experimental group was significantly greater than their control group $(\mathrm{P}<$ 0.001) (18). Their findings were in line with the findings reported by Bastami et al. (2014) (23). Other studies also investigated the effects of green tea, pineapple, turmeric, and Silybum marianum extracts on wound healing.

Based on our findings and also the findings reported by other studies, grape seed extract has antioxidant, antihistaminic, anti-allergic, and anti-inflammatory effects and can boost immunity. Moreover, as a rich source of phenolic compounds such as gallic acid, catechin, resveratrol and a wide spectrum of proanthocyanidins (24), grape seed extract can enhance the volume of collagen fibers and facilitate skin wound healing. Moreover, phenolic compounds (such as epigallocatechin gallate) are determining factors behind the proliferation and the differentiation of keratinocytes (25). In addition to phenolic compounds, grape seed also contains tannins and vitamin E, which can accelerate wound healing (12). It also helps vitamin $C$ enter body cells and hence, it can strengthen membrane of the cells and protect cells against injuries induced by oxidation (14).

In the present study, we attempted to enhance the credibility of the findings through recruiting an adequate sample, using a standard and valid wound assessment tool, doing our best to blind different parties, managing intervening variables, and making telephone contact with the participants to help them adhere to the treatment. Nonetheless, the study had some limitations. For instance, we had no strict control over the participant's personal hygiene. Moreover, the candidates differed from each other regarding factors such as wound healing potential, type of nutrition, and degree of personal mobility. These factors can affect wound healing. However, we could not control their probable effects. On the other hand, study participants' sociocultural, religious, educational, and family backgrounds might have affected their adherence to the treatment. We attempted to minimize the effects of these variables through random allocation.

\subsection{Conclusions}

The findings of the present study indicate that $5 \%$ ointment of grape seed extract can accelerate CS wound healing and reduce its closure time. Therefore, the ointment can be used as a simple and easy-to-access modality to accelerate wound healing and cut the costs of wound management. Besides, it improves women's self-care ability in CS wound management and thereby, strengthens motherchild relationship, reduces the length of treatments, and alleviates wound-related concerns.

\section{Acknowledgments}

This article was part of a master's thesis in nursing, which had been approved by Birjand faculty of nursing and midwifery, Birjand, Iran (the approval code: 9309). Hereby, we thank the research council of the faculty and the research administration of Birjand University of Medical Sciences, Birjand, Iran. We are also thankful to the research unit of Dineh Iran pharmaceutical company, for producing the ointments. We also gratefully thank the dean and staffs of the delivery room of Valiasr hospital as well as all women who agreed to participate in the study. Finally, we appreciate the help of Samaneh Nakhaee, Mohammad BagherRoozgar, and Seyed Alireza Saadatjoo, who reviewed and commented on the study.

\section{References}

1. James D, Philip J, Steer P, Weiner C, Gonik B. High risk pregnancy. UK: Harcoart brace and company; 1999.

2. Rahmanian K, Ghasvari M, Rahmanian V. Cesarean, ever to need attention: prevalence and causes of cesarean section in Jahrom, 1387.J Jahrom Univ Med Sci. 2011;9(1):46-54

3. Jouhari SH, Bayati S, Kheirabadi A, Poor F, Moradi E. Cesarean Section Rate and Its Cause in Fasa in the Year 2011. Journal of Fasa University of Med Sci. 2014;4(3):295-300.

4. Wilson JA, Clark JJ. Obesity: impediment to postsurgical wound healing. Adv Skin Wound Care. 2004;17(8):426-35. [PubMed: 15492679].

5. Schneid-Kofman N, Sheiner E, Levy A, Holcberg G. Risk factors for wound infection following cesarean deliveries. Int J Gynaecol Obstet. 2005;90(1):10-5. doi: 10.1016/j.ijgo.2005.03.020. [PubMed: 15913620].

6. Koigi-Kamau R, Kabare LW, Wanyoike-Gichuhi J. Incidence of wound infection after caesarean delivery in a district hospital in central Kenya. East Afr Med J. 2005;82(7):357-61. [PubMed: 16167709]. 
7. De Vivo A, Mancuso A, Giacobbe A, Priolo AM, De Dominici R, Maggio Savasta L. Wound length and corticosteroid administration as risk factors for surgical-site complications following cesarean section. Acta Obstet Gynecol Scand. 2010;89(3):355-9. doi: 10.3109/00016340903568175. [PubMed: 20199351].

8. Heidari T, Roozbahani N, Farahani L, Attarha M, Torkestani N, Jamilian $\mathrm{M}$, et al. Does Iranian Astragalus gossypinus honey assist in healing caesarean wounds and scars?. Eur J Integrat Med. 2013;5(3):226-33.

9. Veisi F, Zangeneh M, Malekkhosravi S, Rezavand N, Hemiari K, Rezaei M. Subcutaneous vicryl suture covering efficacy on wound complications after cesarean section. J Kermanshah Univ Med Sci. 2011;15(3).

10. Moslemi HR, Jamshidi K, Tavangar MM, Kafshdouzan K. Study of the Effects of Urtica dioica Ointment on the Coetaneous Wound Healing in Cattle. J Med Plants. 2012;2(42):177-82.

11. Hsu S. Green tea and the skin. JAm Acad Dermatol. 2005;52(6):1049-59. doi:10.1016/j.jaad.2004.12.044. [PubMed: 15928624].

12. Kahkonen MP, Hopia AI, Vuorela HJ, Rauha JP, Pihlaja K, Kujala TS, et al. Antioxidant activity of plant extracts containing phenolic compounds. J Agric Food Chem. 1999;47(10):3954-62. [PubMed: 10552749].

13. Salari A, Habibi-Najafi MB, Farhoosh R, Marashi SH. Grape (Vitis vinifera) seed extraction with different solvent systems and assay of antioxidant and antiradical properties. Iran Food Sci Technol Res J. 2009;5(1):1-10.

14. Montaseri M, Ezadi Z, Ezadi M, Sohrabi M. Examine some quality characteristics of grape seed oil and its effects on human health. The second national Conference on Functional Food (Amalgar). Tehran. .

15. Khanna S, Venojarvi M, Roy S, Sharma N, Trikha P, Bagchi D, et al. Dermal wound healing properties of redox-active grape seed proanthocyanidins. Free Radic Biol Med. 2002;33(8):1089-96. [PubMed: 12374620].

16. Hojati , Kazemi K, Tanideh N, Syvani E, Roushan N. The effect of egg yolk and gum tragacunt ointment on wound healing: An experimental study on rabbits. J Med Res. 2004;2(2):33-8.
17. Magann EF, Chauhan SP, Rodts-Palenik S, Bufkin L, Martin JJ, Morrison JC. Subcutaneous stitch closure versus subcutaneous drain to prevent wound disruption after cesarean delivery: a randomized clinical trial Am J Obstet Gynecol. 2002;186(6):1119-23. [PubMed:12066083].

18. Sehhati Shaffaie F, Rashidi Fakari F, Javadzadeh Y, Ghojazadeh M. Ef fect of the phenytoin cream on episiotomy healing in primipara women. SSUJ. 2012;20(2):152-8.

19. Hemmati AA, Foroozan M, Houshmand G, Moosavi ZB, Bahadoram $M$, Maram NS. The topical effect of grape seed extract $2 \%$ cream on surgery wound healing. Glob J Health Sci. 2015;7(3):52-8. doi: 10.5539/gjhs.v7n3p52. [PubMed: 25948437].

20. Hemmati AA, Aghel N, Rashidi I, Gholampur-Aghdami A. Topical grape (Vitis vinifera) seed extract promotes repair of full thickness wound in rabbit. Int Wound J. 2011;8(5):514-20. doi: 10.1111/j.1742481X.2011.00833.x. [PubMed: 21816000].

21. Nayak BS, Ramdath DD, Marshall JR, Isitor GN, Eversley M, Xue S, et al. Wound-healing activity of the skin of the common grape (Vitis Vinifera) variant, Cabernet Sauvignon. Phytother Res. 2010;24(8):11517. doi: 10.1002/ptr.2999. [PubMed: 20066659].

22. Khanna S, Roy S, Bagchi D, Bagchi M, Sen CK. Upregulation of oxidantinduced VEGF expression in cultured keratinocytes by a grape seed proanthocyanidin extract. Free Radic Biol Med. 2001;31(1):38-42. [PubMed: 11425488].

23. Bastami A, Davari Dehkordi N, Ziagham S. The Effect of phonation cream on cesarean wound healing. Iran J Obstet Gynecol Infertil. 2014;17(103):14-20.

24. Saedi Z, Behbahan M. Evaluation of methanol extracts activity of seed skin, leaf and juice from five Iranian grape cultivars on lymphocyte proliferation.J Res Med. 2014;37(4):200-4.

25. Golmakani N, Rabiei ME, Tara F, Asili J, Shakeri MT. The effects of turmeric (Curcuma longa L) ointment on healing of episiotomy site in primiparous women. Iran JObstetr Gynecol Infertil. 2009;11(4):29-39. 